\title{
Effect of bilateral anterior cingulate damage in cats
}

\author{
JUAN A. GOMEZ AND FRED A. METTLER \\ From the Department of Neurosurgery, Montefiore Hospital, New York, N.Y., and the \\ Departments of Anatomy and Neurology, Columbia University, New York, N.Y.
}

Attention has been focused in recent years on the anterior part of the cingulate gyrus, Brodmann's area 24. Smith (1944), Ward (1948), and Glees, Cole, Whitty, and Cairns (1950) independently described loss of fear, reduction of aggressiveness, and marked tameness after removal of the rostral portion of the cingulate gyrus in experimental animals. Such behavioural changes seemed to confirm the debated theory of emotions of Papez (1937) but the application of these implied principles by neurosurgeons has resulted in contradictory data (Le Beau and Pecker, 1950; Livingston, 1953; Siris, 1951; Whitty, Duffield, Tow, and Cairns, 1952; Le Beau, 1954).

Le Beau recommended 'anterior pericallosal topectomy' for epileptic agitation (Le Beau and Pecker, 1950; Le Beau, 1954). Whitty et al. (1952) did not find dramatic changes in behaviour, personality, or autonomic functions after cingulectomy in psychotics but declared it beneficial in neurosis although they recognized that such patients respond easily to other forms of therapy, such as psychotherapy. Livingston (1953) observed early postoperative pleasantness and absence of hostility and fear but was struck by the relatively long period over which many of his patients might continue to change.

At a later date the three original papers by Smith (1944), Ward (1948), and Glees et al. (1950) were challenged by Pribram and Fulton (1954) in a careful experimental study of cingulectomy in monkeys which led them to state that such ablation 'does not lead to the profound and prolonged alterations in behaviour reported in earlier studies'.

In cats Kennard (1955a and b) has reported findings after bilateral cingulectomy closely parallel to the description of cats with extensive caudate lesions and which have been published by another group of workers (Mettler and Mettler, 1942). The present study was designed to re-evaluate gross behavioural changes in cats after the removal of the anterior part of the cingulate gyrus.

\section{METHODS}

Eighteen adult cats were utilized in the present project. After 10 to 15 days of observation for individual characteristics of behaviour, the animals were operated on under aseptic conditions under Nembutal anaesthesia.

After incision of the scalp and pericranial muscle, the anterior part of the temporal muscles was retracted to the sides. In all the animals a trephine hole was made on the right side of the coronal suture and the craniectomy extended across the midline to a symmetrical point on the left side. The dura was cut bilaterally and the brain exposed. The anterior part of both lateral gyri appeared under the exposure. Some of the superior cerebral veins that drain into the longitudinal sinus were cut. The anterior cerebral artery and lateral gyri were gently retracted to visualize the cingulate gyrus on each side and these were ablated. In order to avoid the hazards of this approach we found it more reasonable in 11 animals to approach the surgical target through a small resection of the anterior fifth of the lateral gyrus. To control the effects of that extra ablation, a group of 12 cats had the anterior part of the lateral gyri removed alone or as part of more extensive resections on the lateral surface of the hemispheres.

After surgery the cats were kept at rest in small individual cages at $78^{\circ} \mathrm{F}$. for 24 hours. All animals were observed and examined daily for 30 to 90 days. After the first 28 days no one could distinguish these cats from normal controls. After sacrifice, the brains were fixed in $10 \%$ formalin, photographed, imbedded in paraffin, and stained by alternate Nissl and Mahon techniques. Each section was observed under the microscope and the anatomical lesion evaluated and reconstructed.

\section{RESULTS}

NEUROLOGICAL CHANGES The day after surgery most of the cats had made a very good recovery and were able to walk, eat, and drink. They engaged in their usual grooming activities. Neurological examination disclosed:

Mydriasis In $50 \%$ of the cats the pupils were markedly dilated but reaction to light was preserved. The mydriasis gradually disappeared within one to three days. At post-mortem examination of the brain it was found to be associated with rostrally located lesions that extended towards the beginning of the olfactory area. All cases in which the olfactory zone was involved had mydriasis, and $75 \%$ of the cats with mydriasis had such involvement. 
Hopping and placing reflexes Impairment of hopping and placing reflexes was a very common finding in our cats. In $65 \%$ of the animals this type of abnormal response was present. Lack of hopping and placing reflexes did not appear to be due to ablation of the cingulate cortex since the underlying projection fibres from the sensorimotor cortex were frequently injured, either by direct surgical trauma or by interference with small arteries. More posteriorly placed lesions did not produce this type of abnormality. Hopping and placing reactions reappeared after about five days. After that time, our cats showed no demonstrable sensorimotor defect.

Disregard of depth In $50 \%$ of the cats a lack of appreciation of depth appeared. An animal placed on a table would go to the edge and then walk out as if the floor were on the same plane. Cats affected to a lesser degree would misjudge the distance. After a few miscalculations some of them were very reluctant to jump out of anything. Animals with 'disregard of depth' had marked difficulty in walking down stairs. After the animals were sacrificed and the anatomical sections made, it was found that such phenomena correlated with inadvertently opening the ventricles. Disregard of depth persisted for an average of four to five days, with a maximum duration of 10 days (cat 2984) following ventricular opening without a deeper lesion but one cat with an accidental lesion in the dorsum of the caudate nuclei (no. 3004) had difficulty in appreciating depth for 17 days.

BeHAVIOURAL CHANGES Bilateral anterior cingulectomy did not produce any gross behavioural abnormalities. Minor changes of questionable significance are described below.

About $40 \%$ of the cats seemed to have a tendency to remain unusually quiet for the first three to five days after the operation, despite the absence of any detectable motor or sensory disability. To what extent this was due to surgical trauma is unknown. On the other hand, one animal (no. 2994) was thought to walk and move about more actively than usual for two days after the ablation. Three of the animals appeared more sociable toward the observer (but unchanged toward other cats); one was considered more aggressive. The lesions in these cats did not differ significantly from one another.

After 20 to 25 days, eight of the animals were thought to have become more active than they had been before operation. This was more common in association with extensive ablations. We interpreted such change in activity as hyperreactivity rather than true hyperactivity, according to the criterion established by Mettler and Mettler (1942).

In contrast to the minor behavioural changes of the majority of the experimental animals, one cat (no. 3004) showed a very striking alteration after the surgical procedure. When placed on the floor of the laboratory, for instance, the animal would immediately start to walk in a straight line with slow, monotonous movements-like an automatonuntil the line of progression led him to the walls of the room or some piece of furniture against which the cat would try to pass through by pushing with the head. If left alone he would eventually stop the struggle but would remain with the head against the wall for some time. A noise, or other auditory stimuli, would usually help him to get away from the wall or corner only to walk straight to another similar situation. Also, it was noticed that the cat had a tendency to follow slow-moving objects that pass the visual fields in the horizontal plane. The animal had difficulty in avoiding pitfalls in the room and only freed himself with considerable clumsiness. This syndrome, which has been previously attributed by Mettler and Mettler (1942) to striatal damage, gradually disappeared in about four days, and from then on no distinction could be noted from the other cats. Histological examination of the brain disclosed small inadvertent lesions in the dorsum of both caudate nuclei.

DESCRIPTION OF ANATOMICAL LESIONS Figure 1 represents a drawing of the photographs of the brains involved in this study plus reconstruction and cross sections based on representative microscopic preparations. The removals were not always symmetrical, but in $91 \%$ of the brains area 24 was significantly affected. In all the cats the anterior part of the cingular area was destroyed, as described by Rose and Woolsey (1948), and all but two had lesions in the anterior limbic region of these authors. In six cats the medial olfactory area was also involved. In 11 cats the anterior thirds of the lateral gyrus and supra-splenial gyrus were damaged, but this lesion alone was without effect in neurological or behavioural parameters as proved in 11 additional cats (not shown in the figure) which were used for control of such decortications.

The subcortical white matter and the adjacent projection fibres from the somatosensory cortex were injured to some degree in the great majority of the animals. This is almost impossible to avoid despite considerable effort to prevent it, because of the difficulty in limiting the ablation to the cortex alone and because of secondary interference with small branches of arteries and venous drainage. Other than hopping and placing defects, symptoms referable to such systems were not conspicuous in our animals. It would seem necessary to bear in mind such subcortical lesions when analysing some of the 

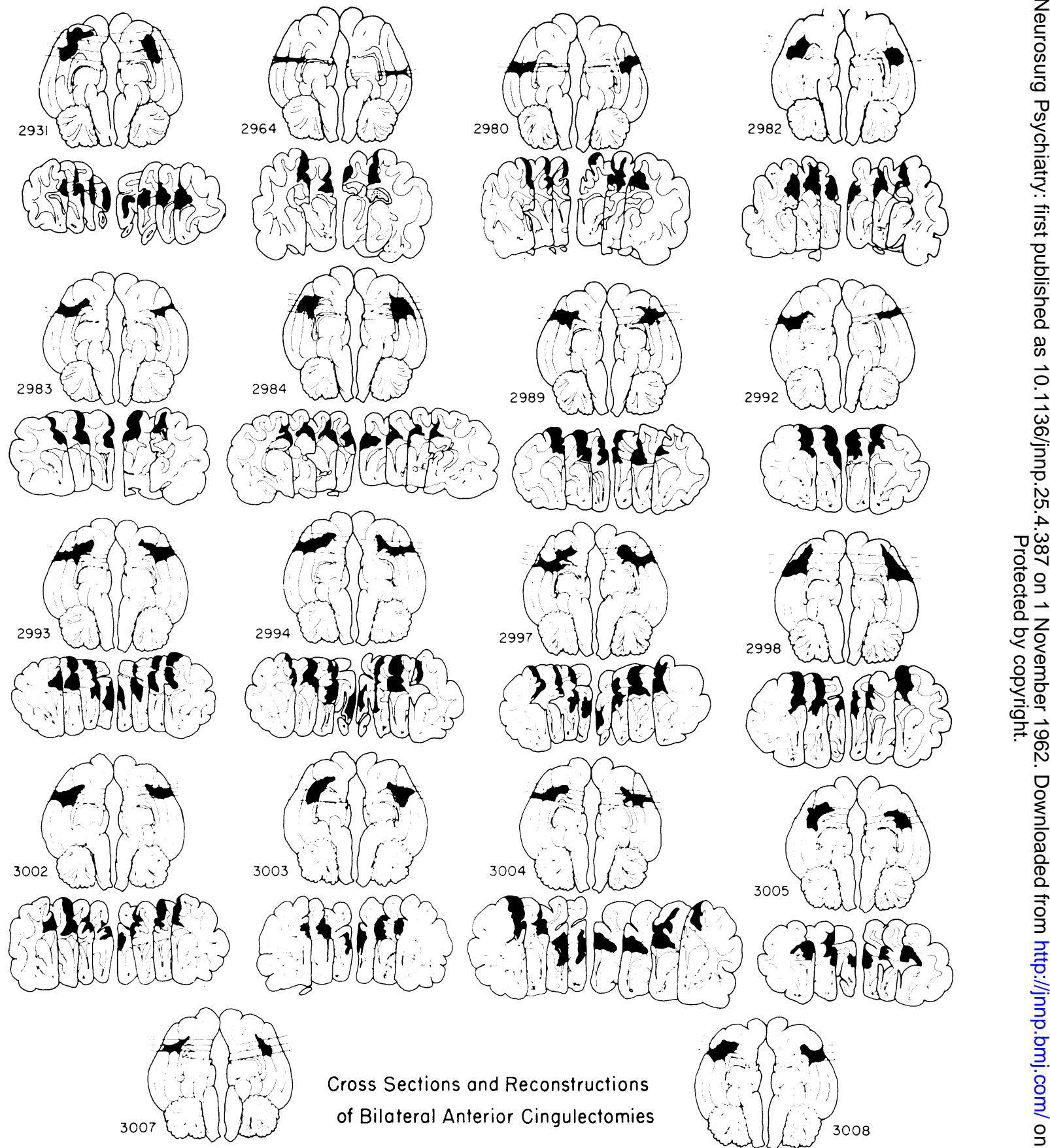

Cross Sections and Reconstructions of Bilateral Anterior Cingulectomies
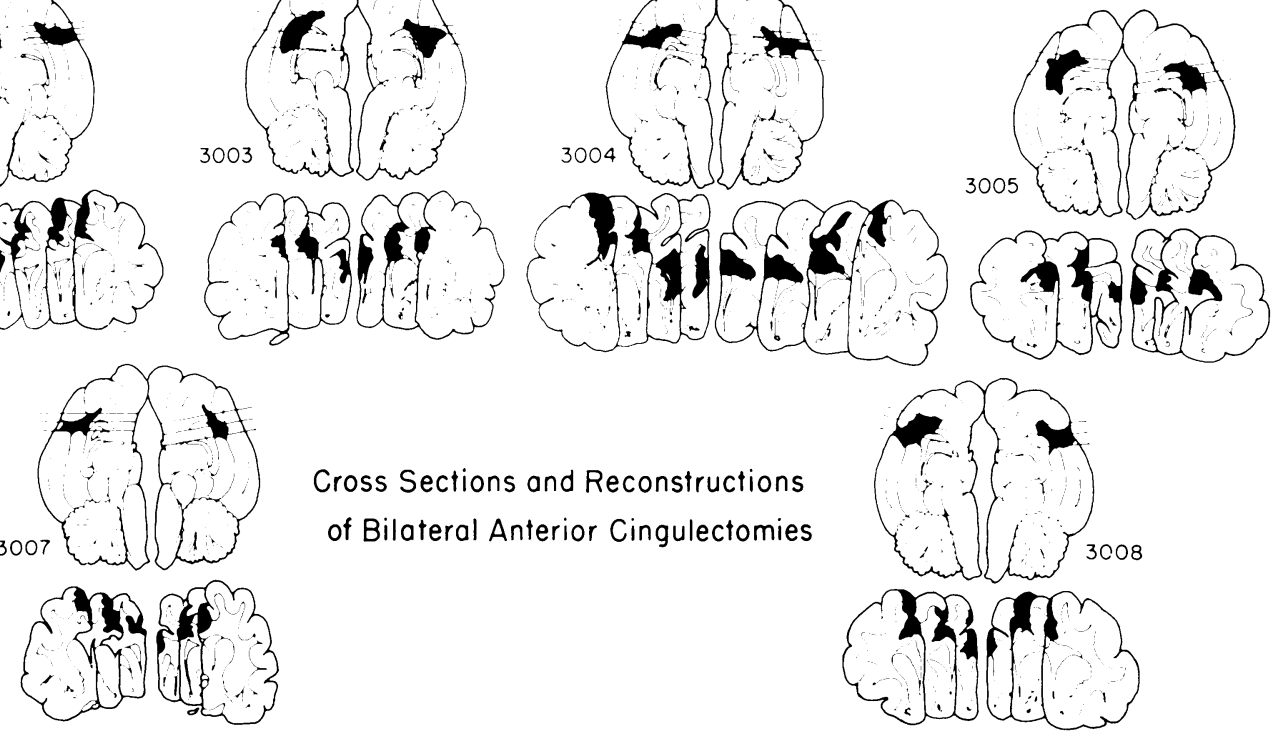

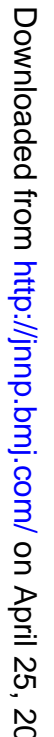

FIG. 1. This figure represents cross sections and reconstructions of bilateral anterior cingulectomies in 18 cats, based on representative microscopic preparations and photographs of the fixed brains. 
results that have been reported in the literature.

In nine cats the ventricles were inadvertently opened and in one the dorsal surface of the caudate was injured. Also, fibres from the corpus callosum were frequently damaged when the adjacent part of the cingulate gyrus was removed.

\section{DISCUSSION}

Our failure to observe any dramatic and persistent changes in the behaviour of cats after destruction of the anterior cingulate gyrus is not in accordance with some reports in the literature. The earlier work was done independently by three authors (Ward, 1948; Glees et al., 1950; and Smith, 1944). About 14 monkeys were used in these experiments. Two were trained animals. The work by Smith (1944) was reported in the Federation Proceedings, and only a short summary seems to be available for study. $\mathrm{He}$ used untrained monkeys which were observed for behavioural and autonomic changes after removal of the cingulate gyrus. Surgery was followed by a period of stupor after which the animals were noted to be less active and to exhibit an increase in vocalization and piloerection. They were less frightened when approached by human beings ('will take food from one's hand') but did not allow themselves to be handled. After a week only tameness was noted.

In 1948 Ward reported the effects of cingulectomy in four monkeys. One brain was examined histologically. The animals lost their pre-operative shyness and fear of man, and their 'social conscience'. They behaved as if other monkeys were inanimate ('walk over them', 'even sit on them'). One animal was hyperactive for two weeks.

Shortly after the report by Ward, Glees and his associates (1950) published a careful study of seven cingulectomized monkeys with complete anatomical studies. Six of the animals had bilateral lesions. One developed hemiplegia after the operation and another had ataxia. In spite of the description given in the article about the behaviour of each monkey (some showed very little change, others an increase in activity) the authors gained the general impression that 'there was an increased tameness and reduction of aggressiveness with apparent loss of the sense of danger' in all but two of their animals. These phenomena were transitory.

Pribram and Fulton (1954) were unable to confirm these reports. They used 20 monkeys and secured anatomical confirmation of all the lesions. In trained monkeys they found no changes in visual discrimination or delayed response and no changes in systematically observed rates of cage activity. The social structure of the colony remained unchanged. Pribram and his colleagues (Pribram and
Weiskrantz, 1957; Mirsky, Rosvold, and Pribram, 1957) subsequently found that cingulectomy resulted in rapid extinction of both pre- and post-operatively acquired conditioned avoidance. The authors also observed that it 'is clear that the unqualified conclusion, on the basis of experiments in monkeys, that the cingulate gyrus is intimately concerned with personality and emotion is unacceptable'.

On the other hand, Kennard (1955a and b) has described a syndrome in cats that bears no relation to other observations in the literature. It is said to be characterized by 'confused, perseverative, obsessive behaviour, and a plastic tendency whereby the animal may be posed for long periods of time'. Also she noted changes in emotional reactions ('rage was easily elicited') contradictory of previous reports. In monkeys, surprisingly enough, the author found an entirely different pattern (the animals became 'soft') more in accord with the conclusions of Smith and Ward. We could not duplicate her results in cats.

Autonomic changes have been reported in connexion with the cingulate gyrus (Smith, 1949; Kaada and Jasper, 1952). Horsley and Schäfer in 1888 were apparently the first to ascribe specific neurological manifestations to lesions in the cingulate gyrus. They thought that anaesthesia was produced. More recently Vonderahe (1943) described apathy in a patient with a cingulate lesion. Later, Nielsen and Jacobs (1951) reported a case with bilateral lesions in the cingulate gyri, and Barris and Schuman (1953) published an article describing 'the syndrome of anterior cingulate gyri' based on two patients, one of their own and the previous report by Nielsen.

Few authorities in the field of clinical neurology have been able to recognize any specific syndrome related to anterior cingulate lesions. Confirmation of the expected changes in personality could not regularly be obtained in human beings treated by cingulectomy and the operation has fallen into general disuse. Those persons who do advocate cingulectomy have described different results and their indications vary from treatment of neurosis to epilepsy. Le Beau and his associates in France have reported good results with anterior pericallosal topectomy in some forms of psychomotor agitation in epileptics (Le Beau and Pecker, 1950; Le Beau, 1954). Whitty et al. (1952) found no worthwhile improvement in psychotics, no change in one patient with depression, but obtained a 'cure' in two out of six neurotic patients. In contrast to the results of the experimental work done with Glees et al. (1950), Whitty's patients showed few autonomic changes and their mental state did not alter dramatically. Tow and Whitty (1953) subsequently reported little change in the total personality picture 
but observed a slight reduction in inhibition. Livingston (1953) was impressed with the relatively long period over which some of his patients continued to change. Negative conclusions were reached by Mettler (1949) and by Scoville, Wilk, and Pepe (1951) in independent studies.

Our observations fully agree with those of Pribram and Fulton (1954) and we have to concur with Pechtel, McAvoy, Levitt, Kling, and Masserman (1958) in their statement that even a preliminary assessment of the function of the cingulate gyrus would be premature. In our hands anterior bilateral simultaneous ablations of cingulate gyri did not produce gross behavioural or neurological deficit. No specific grossly-observable syndrome can be ascribed to that area.

\section{SUMMARY AND CONCLUSIONS}

In 18 adult cats the cingulate area was removed bilaterally and the animals were observed for at least 35 days. Twelve cats with ablation of the anterior third of the lateral gyri were used as controls. All animals were sacrificed and the brains were photographed, fixed, stained, and examined microscopically for anatomical reconstruction of the lesions.

No gross behavioural disturbance and no specific neurological syndrome could be attributed to destruction of the cingulate area alone.

\section{REFERENCES}

Barris, R. W., and Schuman, H. R. (1953). Neurology (Minneap.), 3, 44.

Glees, P., Cole, J., Whitty, C. W. M., and Cairns, H. (1950). J. Neurol. Neurosurg. Psychiat., 13, 178.

Horsley, V., and Schäfer, E. A. (1888). Phil. Trans. B., 179B, 1.

Kaada, B. R., and Jasper, H. (1952). A.M.A. Arch. Neurol. Psychiat., $68,609$.

Kennard, M. A. (1955a). J. nerv. ment. Dis., 121, 34.

- (1955b). J. Neurophysiol., 18, 159.

Le Beau, J. (1954). Psycho-Chirurgie et Fonctions Mentales. Masson, Paris.

—, and Pecker, J. (1950). Semaine Hôp. (Paris), 26, 1536.

Livingston, K. E. (1953). Res. Publ. Ass. nerv. ment. Dis., 1951, 31, 374.

Mettler, F. A., and Mettler, C. C. (1942). Brain, 65, 242.

(1949). Selective Partial Ablation of the Frontal Cortex (case no. 40). Edited by F. A. Mettler, pp. 41, 75, 415, 436. Hoeber, New York.

Mirsky, A. F., Rosvold, H. E., and Pribram, K. H. (1957). J. Neurophysiol., 20, 588.

Nielsen, J. M., and Jacobs, L.. L. (1951). Bull. Los Angeles neurol. Soc., 16, 231.

Papez, J. W. (1937). Arch. Neurol. Psychiat. (Chic.), 38, 725.

Pechtel, C., McAvoy, T., Levitt, M., Kling, A., and Masserman, J. H. (1958). J. nerv. ment. Dis., 126, 148.

Pribram, K. H., and Fulton, J. F. (1954). Brain, 77, 34.

, and Weiskrantz, L. (1957). J. comp. Physiol. Psychol., 50, 74.

Rose, J. E., and Woolsey, C. N. (1948). J. comp. Neurol., 89, 279.

Scoville, W. B., Wilk, E. K., and Pepe, A. J. (1951). Amer. J. Psychiat., 107,730 .

Siris, J. H. (1951). Psychiat. Quart., 25, 251.

Smith, W. K. (1944). Fed. Proc., 3, 42.

(1949). Trans. Amer. neurol. Ass., 74, 169.

Tow, P. M., and Whitty, C. W. M. (1953). J. Neurol. Neurosurg. Psychiat., 16, 186.

Vonderahe, A. R. (1943). Ohio State med. J., 39, 325.

Ward, A. A., Jr. (1948). J. Neurophysiol., 11, 13.

Whitty, C. W. M., Duffield, J. E., Tow, P. M., and Cairns, H. (1952). Lancet, 1, 475. 